\title{
EN TORNO AL CONCEPTO DE CUERPO DESDE ALGUNOS PENSADORES OCCIDENTALES
}

\section{Around the Body's Concept from Some Western Thinkers}

\author{
José Arlés Gómez Arévalo \\ Asseneth Sastre Cifuentes*
}

Recibido: 27 de marzo de 2008 • Revisado: 6 de mayo de 2008 - Aceptado: 23 de mayo de 2008

\begin{abstract}
Resumen
El presente artículo de revisión pretende aportar a la reflexión sobre el concepto de cuerpo desde la historia de las ideas filosóficas que en Occidente han venido sustentando visiones paradigmáticas al respecto, para lo cual los autores incursionan en las escuelas o corrientes que han hecho alusión al tema desde el mundo de la filosofía. Este trabajo hace parte no sólo de la plataforma epistemológica del grupo de investigación Cuerpo, Sujeto y Educación que integran los autores, sino que también constituye un aporte a la fundamentación de cualquier trabajo comprometido con las concepciones del cuerpo y lo corporal, así como un marco necesario de cualquier propuesta programática que sitúe al cuerpo como foco de reflexión, conceptualización e intervención, por cuanto ofrece diversas comprensiones sobre el cuerpo y lo corporal, que de manera explícita o implícita soportan la generación de lineamientos pedagógicos, curriculares, investigativos, interventivos, así como la legitimación de diversas prácticas sociales.
\end{abstract}

\section{Palabras clave}

Cuerpo humano, filosofía, mente, espíritu, pensamiento, realidad, fenómeno.

Docentes-investigadores Departamento de Humanidades y Facultad de Cultura Física, Deporte y Recreación de la Universidad Santo Tomás, Bogotá. Correos electrónicos: intiyang@hotmail.com, mariasastre@correo.usta.edu.co. 


\begin{abstract}
The present revision article seeks to contribute to the reflection on the body concept from the history of the philosophical ideas that in the west have come sustaining paradigmatic visions regarding the body itself, to which the authors foray into the schools or disciplines that have made allusion to the topic from the philosophy's world. This work does not only make part of the platform epistemological platform of the group of investigation Body, Subject and Education that the authors integrate, but rather it constitutes a contribution to the foundation of any committed work with the conceptions of the body and the corporal thing, as well as a necessary framework of any programmatic proposal that locates the body like reflection focus, conceptualization and intervention, as long as it offers diverse understandings on the body and the corporal thing, that support in an explicit or implicit way the generation of pedagogic, curricular, investigative policies, as well as the legitimating of diverse social practices.
\end{abstract}

\title{
Key words
}

Human body, philosophy, mind, spirit, thought, reality, phenomenon.

\section{Introducción}

Frente a la invitación de elaborar una reflexión en torno al concepto de cuerpo, en estudios anteriores, se ha sostenido (Gómez \& Sastre, 2004) que es en el cuerpo donde la existencia humana adquiere una dimensión espacio-temporal, y es éste el que hace al ser humano parte activa de la naturaleza y del proceso de la vida. Es allí donde se integran sus múltiples experiencias y manifestaciones y donde se sintetizan, en una relación de interdependencia sus diferentes dimensiones: biológica, cognoscitiva, psicomotriz, emocional, relacional y comunicacional, ética, estética y trascendente. Por otra parte, se ha sostenido, desde una perspectiva neohumanística, que una cultura del cuerpo contemporánea puede ser entendida como un fundamental aprendizaje y construcción de sí mismo, como cultivo de valores básicos de expresión personal y de relación social a través de las experiencias corporales. Dichas experiencias son elaboradas por el ser humano en lo que podríamos llamar el "arte de vivir el cuerpo". Estas elaboraciones provienen del cuerpo, se dan a través de y para el cuerpo cuyo propósito es la expresión de su pensamiento, sentimiento y movimiento (acción) en relación consigo mismo, con los otros y con su medio ambiente vital.
Más allá de esta comprensión situada en una reflexión individual, cualquier trabajo comprometido con las concepciones del cuerpo y lo corporal debe partir del reconocimiento de la historia de las ideas filosóficas que han venido sustentando visiones paradigmáticas con respecto a éste, reconocimiento que demanda incursionar en las escuelas o corrientes que han hecho alusión al tema desde el mundo de la filosofía. Cualquier propuesta programática que sitúe al cuerpo como foco de reflexión, conceptualización y eje de la intervención requiere, por otra parte, de una claridad epistemológica que provea el soporte necesario a las comprensiones sobre el cuerpo y lo corporal a partir de las cuales, de manera explícita o implícita, se generan lineamientos pedagógicos, curriculares, investigativos, interventivos, y se legitiman ciertas prácticas sociales.

\section{Punto de partida}

Así como las ciencias experimentales hacen una descripción del cuerpo como realidad física, tangible y medible, descripción básica a la hora de hablar de éste y en el que desempeñan un papel relevante todas las disciplinas que describen procesos relacionados con lo corporal y lo mental: biología, informática, 
ciencia cognitiva, cibernética, lingüística, medicina, farmacología, psicología, física, química, matemáticas, etc., asimismo, la historia del pensamiento filosófico evidencia una notoria preocupación por el análisis sobre la problemática del cuerpo humano.

Dicha problemática ha constituido un aspecto fundamental dentro del contexto de la filosofía, especialmente en la época contemporánea. En esta última época, se debe, particularmente, a la orientación fenomenológico-existencial, la cual ha descubierto la fecunda noción de "cuerpo propio" y planteado el problema del cuerpo -de una manera original y sistemática- en el centro de las preocupaciones dominantes del pensamiento, especialmente en la época contemporánea'.

\section{Aportes a la reflexión sobre el cuerpo en algunos pensadores occidentales}

\section{Antigüedad}

En el mundo griego y hebreo, el término soma ${ }^{2}$ no hace alusión propiamente al cuerpo como contraposición al alma, sino que hace referencia al cadáver. Es más, el cuerpo no se concibe como una "unidad de partes" armónicas, sino como una yuxtaposición de órganos y elementos separados. En la Biblia se habla del término ruah (aliento cargado de vapor), que será traducido a pneuma (ráfaga de viento) en el idioma griego, más adelante se traducirá por spíritu

1 En tal sentido, el concepto central, que de inmediato se impone en la fenomenología francesa, es el de cuerpo "propio", "sujeto" o "fenomenológico", concepto clave que sintetiza acabadamente la renovada visión de la esencia del hombre respecto al tradicional problema metafísico del cuerpo y el alma, para el cual permanece subestimada la realidad (corporeidad), oculto el fenómeno (corporalidad) e indiferente al misterio (encarnación) del cuerpo humano.

2 Proviene del griego somatikós, que se deriva de soma (cuerpo). Hace alusión al cuerpo, al igual que al efecto que pueden producir en el cuerpo ciertos estados emocionales o psíquicos, de igual manera se relaciona con otras raíces griegas como el término compuesto "psicosomático". en latín, para referirse a aquello que se diferencia de lo corporal ${ }^{3}$.

En las politeias de las ciudades-Estados griegas, Platón y Aristóteles ${ }^{4}$ recogen la tradición en sus obras centrales dedicadas a la organización del Estado. El ciudadano debería formarse para responder a los requerimientos del Estado y en gran medida se debía a él. Uno de los aspectos a los cuales se prestó mayor atención fue precisamente a la "formación del cuerpo" pues se consideraba que la robustez corporal era una condición necesaria para seguir el proceso de formación continua, pues en los cuerpos débiles no se gestaban almas heroicas y guerreras.

Entre los griegos hay un reconocimiento muy claro de la ineludible dinámica animal de la especie, que antes que nada la hace biológica, sujeta a los ritmos naturales de una corporalidad sobre la cual se construye el espíritu y el alma mediante una consciente labor pedagógica. De allí la importancia del pedagogo. En esta parte es importante el rastreo de los documentos de Platón (La República) y Aristóteles (La Política), además, de algunos documentos que mencionen a los filósofos de las escuelas estoica (Epicteto) y hedonista (Epicuro de Samos), quienes con sus ideas encontradas sobre las virtudes (estoicos) y los placeres (hedonistas) hicieron un aporte a la manera como se entendió el cuerpo y lo corporal en Grecia y la influencia que tuvieron en los desarrollos filosóficos posteriores.

3 Cf. Fisogni, Vera, Il corpo dello spirito, citado en: http: //serbal. pntic.mec.es/ cmunoz11/vera.pdf.

4 Aristóteles define el alma: "La entelequia primera de un cuerpo natural que tiene la vida en potencia", principio en movimiento, de crecimiento, de generación, unifica todas sus funciones, sin exceptuar las operaciones de la sensibilidad y del entendimiento. Para Aristóteles, el alma no puede subsistir sin un cuerpo. En pocas palabras el alma no es esa exiliada de que habla Platón, encerrada en un cuerpo con la nostalgia de despojarse para siempre de el; es ella la que asegura la armonía funcional de las funciones vitales. 
Para Platón, si el alma sobrevive a la muerte del cuerpo, ha de ser algo diferente de éste ${ }^{5}$. En la medida en que el destino del alma no es el mundo corporal y sus valores sino el mundo espiritual, ya que ambos mundos están enfrentados, la tarea moral, religiosa e intelectual del hombre consistirá en intentar liberarse de las exigencias del cuerpo y de sus limitaciones.

En Aristóteles, su concepción sobre el cuerpo presenta dos etapas: en el primer periodo de su reflexión filosófica se inclinaba hacia el dualismo, compartiendo las tesis antropológicas de Platón, así lo atestiguan ciertos trozos de sus obras perdidas, como el estudio de Nuyens, "La Evolución de la Psicología de Aristóteles", publicado en Lovaina en 1948. En la segunda etapa, el filósofo griego da cuenta de cómo los organismos vivos se pueden explicar filosóficamente mediante la teoría general del hilemorfismo: todo ser material está compuesto de materia y forma. La materia no existe sin una forma determinada, la forma no existe más que como forma de un determinando ser material. En lugar de materia y forma, Aristóteles recurre a la terminología cuerpo-alma. La forma sustancial de un organismo vivo se llama psique o alma, el cuerpo es la materia viva e informada por la psique. En este pensador, el cuerpo puede entonces tener dos significados diversos: a veces es la materia que entra en el organismo vivo y es informada por el alma, otras

5 Dice Platón: "La razón no tiene más que un camino que seguir en sus indagaciones; mientras tengamos nuestro cuerpo, y nuestra alma esté sumida en esta corrupción, jamás poseeremos el objeto de nuestros deseos; es decir, la verdad. En efecto, el cuerpo nos opone mil obstáculos por la necesidad en que estamos de alimentarle, y con esto y las enfermedades que sobrevienen, se turban nuestras indagaciones. Por otra parte, nos llena de amores, de deseos, de temores, de mil quimeras y de toda clase de necesidades; de manera que nada hay más cierto que lo que se dice ordinariamente: que el cuerpo nunca nos conduce a la sabiduría. Porque, ide dónde nacen las guerras, las sediciones y los combates? Del cuerpo con todas sus pasiones" (2001, p. 15). veces indica propiamente el organismo vivo (materia más forma $)^{6}$.

Claude Tresmontant (1974), en su obra El problema del alma, va a indicar que en Aristóteles un cuerpo organizado está compuesto por una materia y por un alma que informa esa materia. No existe problema alguno en cuanto a las relaciones entre alma y cuerpo, ya que el cuerpo vivo es la misma alma en cuanto que informa una materia.

En el mundo semita, fuente de primer orden para la cosmovisión cristiana, no se conoce propiamente el dualismo entre cuerpo y alma ${ }^{7}$. Es cierto que las traducciones a lenguas vernáculas de la Biblia dan cuenta de términos parecidos a la connotación dualista entre cuerpo y alma. La antropología semita interpreta al hombre como unidad. Es más, la idea de una "caída" del alma preexistente en un cuerpo, según Platón, es desconocida en esta cultura. La existencia corporal y física, en la tradición hebrea, nunca ha sido considerada como culpable o pecaminosa, vergonzosa o impura, por el contrario, hasta las expresiones más

6 Admitiendo la existencia de la forma y la materia aristotélicas (hilemorfismo) y suponiendo que el alma hace en el hombre el papel de forma y el cuerpo de materia, se podría comprender también esa interacción eficiente del alma y cuerpo, puesto que serían dos principios que por su misma naturaleza están llamados a fundirse y actuar unidos. El hombre es concebido por Aristóteles de un modo hilemorfístico, es decir, como un compuesto de materia y forma. El cuerpo funciona como materia prima y el alma como forma sustancial. La unión existente entre alma y cuerpo es una unión sustancial. Si la unión accidental supone básicamente que los elementos unidos existen ya constituidos antes de la unión, la unión sustancial, por el contrario, constituye esos elementos y ella misma los hace existir. En la accidental, los elementos unidos persisten como siendo distintos y existiendo paralelamente; en la sustancial se fusionan en una unidad única. En ella alma y cuerpo marchan juntos en una unidad de operación, forman un único ser.

7 En la Biblia, el alma (nephes) es el "soplo de vida" que hace del hombre un "alma viviente", pero este término es muchas veces empleado en contraste con el mundo celeste de Dios y del espíritu (ruah). Entonces se debió completar la dicotomía filosófica (cuerpo-alma) con la tricotomía teológica (cuerpo-alma-espíritu). Esta última vino a ser tradicional en Oriente, y también se nota una cierta confusión en el uso y en el significado de los términos. 
humanas como la sexualidad y la fecundidad son consideradas de gran valor y tenidas como bendición divina (Tresmontan, 1974, p. 55).

El mismo término "basar" fue traducido por sarx (carne, a veces cuerpo). La carne no significa cuerpo en oposición al alma, sino que significa la totalidad corpóreo-espiritual del hombre, como unidad psicofísica. También el término carne indica parentesco social y familiar: nuestra carne significa nuestro hermano. Toda carne significa todos los hombres, sin oposición al espíritu .

\section{Edad Media}

Con el advenimiento del cristianismo como religión oficial del Imperio Romano, el trabajo filosófico se vio necesariamente impregnado del elemento religioso. Las traducciones de los filósofos griegos que hicieran los filósofos árabes cayeron en manos de los pensadores cristianos, quienes vieron la necesidad de fundamentar filosófica y teológicamente al cristianismo. Las ideas de Plotino (Henry, 1932, pp. 707-925) (discípulo de Platón), de Aristóteles, quien consideró al cuerpo como "cierto instrumento natural del alma", y

$8 \quad$ El término hebreo ruah, aparece 378 veces en todos los periodos del idioma bíblico y tiene sus equivalentes en ugarítico, arameo y arábigo, se usa comúnmente como: hálito, aire, viento, brisa, coraje, temperamento. Para su uso como compuesto antropogónico, el espíritu era un motor impersonal invisible, sin identidad, que potenciaba y sustentaba un cuerpo en movimiento junto con la circulación sanguínea; cuando estos cesaban, no se depositaban en ningún sitio fáctico sino que despojan al alma viva de su movilidad y la convertían en un ser inerte, quieto, reducido a su mínima acción. El espíritu, luego de la muerte, por su lado vuelve a Dios, o simbólicamente a su fuente generadora (Eclesiastés 12: 7).

$9 \quad$ Plotino es el representante principal y más genuino de la filosofía neoplatónica. El alma humana, en este pensador, es emanación inmediata del Alma universal, es anterior y posterior al cuerpo en su ser y en sus operaciones. Esta preexistencia del alma con respecto al cuerpo, lleva consigo la independencia y superioridad de ésta en su ser y en sus funciones, independencia que, no sólo comprende las funciones de la parte superior e intelectual, sino también de la parte inferior; de manera que el alma es completamente activa e independiente del cuerpo y de sus órganos en la sensación, lo mismo que en la intelección. del mismo Platón fueron adaptadas a las necesidades del cristianismo ${ }^{10}$.

El concepto del cuerpo como "cárcel del alma", acuñada por Platón y recogida por Plotino, tendrá acogida en algunos pensadores cristianos. Según este pensador se explica así: "Si el alma es sustancia, será una forma separada del cuerpo o, para decirlo mejor, será lo que se sirve del cuerpo". Esta frase sirvió de base junto con otras ideas filosóficas para hacer toda una disertación sobre el maltrato de lo físico para alcanzar la liberación del espíritu. La mortificación y la tortura del cuerpo fueron parte de una tradición espiritual cristiana, empeñada en doblegar los impulsos de la naturaleza biológica, en búsqueda de una perfección del alma para la cual el cuerpo era un lastre. Éstas y otras ideas se pueden rastrear en algunos pensadores de la Edad Media que hicieron aportes desde sus cosmovisiones religiosas y filosóficas.

Tomás de Aquino entendió al hombre como totalidad y dijo sobre el cuerpo que el fin próximo del mismo es el alma racional y las operaciones de ella. Pero la materia es en vista de la forma y los instrumentos en vista de las acciones del agente. También el cuerpo es materia del alma y el alma es a su vez la forma del cuerpo. La materia no recibe la forma a no ser causa del fin. Por eso el fin es la causa de las causas, porque todo lo que obra tiende siempre a algo. En el maestro de Aquino, la relación del alma y el cuerpo es una relación natural, no una situación forzada y antinatural, según la cual estaría el alma en el cuerpo como el prisionero en la celda. No se puede interpretar la interdependencia entre el alma y el cuerpo como un castigo para el alma, en contra de lo que los neoplató-

10 Los antiguos Padres de la Iglesia insisten siempre en la unidad del alma y el cuerpo; el hombre entero es una sustancia y, por otra parte, el alma existe ya por sí misma y anima el cuerpo que tiene su consistencia fuera de ella. Es necesario admitir que los esfuerzos de los antiguos Padres por hacer inteligible la unidad del alma y del cuerpo no permiten, desde el punto de vista especulativo, captar esta unidad en su esencia. El problema, más tarde, será más fácil para los autores escoláticos que consideran el alma como la "forma" de un ser viviente orgánico. Cf. Spidlík, Tomas, Antropología cristiana: cuerpo, alma, espíritu, citado en: http: // members.fortunecity.es/mariabo/la_antropologia_cristiana.htm. 
nicos afirmaban, y que dio pie al desarrollo de algunas herejías basadas o inspiradas en el gnosticismo, como la de los cátaros ${ }^{11}$.

Así como Aristóteles había concebido la existencia de una sola alma en el ser humano, que engloba las funciones vegetativa y sensitiva, santo Tomás afirma que esa única alma es la que regula todas las funciones del "hombre" y determina su corporeidad:

Es evidente, por otra parte, que lo primero por que el cuerpo vive es el alma, y como la vida se manifiesta por operaciones diversas en los diversos grados de los seres vivientes, aquello por lo que primariamente ejercemos cada una de estas funciones vitales es el alma. Ella es, en efecto, lo primero que nos hace nutrirnos y sentir y movernos localmente, como también entender. Este primer principio de nuestro entendimiento, llámasele entendimiento o alma intelectiva, es, por tanto, la forma del cuerpo, y esta demostración es de Aristóteles en el tratado Del alma, lib. 2, tex. 24 (de Aquino, 1988, p. 23).

Pese a la lucidez y claridad de filósofos como Tomás de Aquino y Alberto Magno ${ }^{12}$, muchas de las ideas medievales transmitidas por los predicadores cristianos permitieron identificar los instintos, las enfermedades, las desgracias y la muerte con el pecado que se ejecutaba con el cuerpo. Según Ch. Frugoni, el cuerpo de la mujer en la Edad Media era casi tan temible como el demonio, pues era su instrumento y de él se servía, para perder a los santos (Frugoni, 1977, pp. 177-188). El logro de la espiritualidad no se entendió dentro del cuerpo sino prescindiendo de

11 También denominados "albigenses". Por su desprecio al cuerpo (que por algo es material), los cátaros se oponían al matrimonio y practicaban una ascesis tan rigurosa que algunos llegaban a morirse literalmente de hambre, dieta a la que llamaban "suicidio de liberación". Fueron condenados como herejes por la Iglesia Católica.

12 Cf. Etienne Gilson, La philosophie au moyen-âge, vol.II: De saint Thomas d'Aquin à Guillaume d'Occam, Payot, Paris, 1922 Según este historiador francés, Alberto Magno, comprendió que la filosofía y la ciencia árabes, que recogían gran cantidad de material de la Grecia clásica perdidos para Occidente, tenían un valor inestimable para los teólogos cristianos. Por ello se propuso, entre otras cosas, "hacer inteligibles a los latinos todas las partes de la filosofía aristotélica". Pero su esfuerzo no se limitó a Aristóteles sino que se extendió a toda la ciencia árabe y judía y al neoplatonismo. él; en algunos momentos de la historia europea, los "carnavales" fueron espacios transitorios, para escapar de esta realidad ${ }^{13}$.

\section{Renacimiento}

Aunque en términos generales no se puede hablar de una "filosofía renacentista", sí hubo un movimiento de ideas religiosas, culturales, políticas y sociales, que permitieron dar un viraje al teocentrismo medieval. El hombre fue puesto en el centro de la creación y, por tanto, se reivindicó al cuerpo y lo corpóreo en el arte: la pintura, la escultura, la música y la literatura son expresiones de este proceso. Es importante rastrear las fuentes de artistas, pensadores y literatos que de alguna manera hicieron alusión al tema del cuerpo y lo corporal, bien sea en sus escritos o sus obras artísticas: Dante Alighieri, Bocaccio, Tomás Moro, Da Vinci ${ }^{14}$, Rafael, Miguel Ángel, Giotto, Giordano Bruno, etc. Con el nuevo sentimiento de ser un individuo, de ser él mismo antes de ser miembro de una comunidad, el cuerpo se convierte en la frontera precisa que marca la diferencia entre un hombre y otro. La estructuración individualista progresa lentamente en el universo de las prácticas y de las mentalidades del Renacimiento.

13 El cuerpo medieval no se distingue del hombre, como sucederá con el cuerpo en la Modernidad, entendido como factor de individuación. Un ejemplo son las fiestas de las altas capas de la sociedad o la Iglesia, que no alteraban el orden existente, tendían a consagrar la estabilidad, la inmutabilidad y la perennidad de las reglas que regían el mundo: jerarquías, valores, normas y tabúes religiosos, políticos y morales corrientes. En cambio, el carnaval, creaba una "segunda vida" era el triunfo de una especie de liberación transitoria, más allá de la órbita de la concepción dominante, la abolición provisional de las relaciones jerárquicas, privilegios, reglas y tabúes. Se oponía a toda perpetuación, a todo perfeccionamiento y reglamentación, apuntaba a un porvenir aún incompleto. "El cuerpo humano es, en las tradiciones populares, el vector de una inclusión, no el motivo de una exclusión (en el sentido en que el cuerpo va a definir al individuo y separarlo de los otros, pero también del mundo); es el que vincula al hombre con todas las energías visibles e invisibles que recorren el mundo".

14 Nicholl recuerda cómo da Vinci, realiza una treintena de disecciones, dejando notas y fichas sobre la anatomía humana. Pero los manuscritos de Leonardo sólo tienen una pequeña influencia en su época y luego permanecen prácticamente en secreto por mucho tiempo. "Oh, tu, que te libras a especulaciones sobre esta máquina nuestra, no te entristezcas porque la conoces a causa de la muerte de otra persona; alégrate, en cambio, de que nuestro creador le haya proporcionado al intelecto tan excelente instrumento" (Leonardo da Vinci) (Nicholl, 2005). 
Limitado en primer término a ciertas capas de sociedad privilegiadas, a ciertas zonas, a ciudades sobre zonas rurales, la persona se diferencia de sus semejantes y trata de configurar su individualidad.

Desplazar la visión teocéntrica conduce al hombre a considerar al mundo que lo rodea como una forma pura, indiferente, una forma vacía que sólo la mano de él, a partir de este momento, puede moldear. Un ejemplo claro en el Renacimiento son los anatomistas, quienes abren el camino para otros descubrimientos, al fisurar, junto a las fronteras del cuerpo, las del mundo terrestre y las del macrocosmos (Pouchelle, 1983). Junto con esta nueva visión del cuerpo humano surge el saber anatómico en la Italia del Quattrocento, principalmente en las Universidades de Padua, Florencia y Venecia. A partir de las primeras disecciones oficiales, a comienzos del siglo XV y, luego, con trivialización de la práctica en los siglos XVI y XVII, se produce uno de los momentos clave del individualismo occidental: antes el cuerpo no era la singularización del sujeto al que le prestaba rostro. El hombre, inseparable del cuerpo no estaba sometido a la singular paradoja de poseer un cuerpo ${ }^{15}$.

\section{Modernidad}

Con la aparición del racionalismo cartesiano ${ }^{16}$ se dio la separación entre cuerpo y conciencia, entre razón

15 Con el nuevo sentimiento de ser un individuo, de ser él mismo, antes de ser miembro de una comunidad, el cuerpo se convierte en la frontera precisa que marca la diferencia entre un hombre y otro. La estructuración individualista progresa lentamente en el universo de las prácticas y de las mentalidades del Renacimiento. Limitado en primer término a ciertas capas de sociedad privilegiadas, a ciertas zonas, a ciudades sobre zonas rurales, el individuo se diferencia de sus semejantes.

16

Un dualismo de este tipo tiene que enfrentarse a serios problemas. Si existe un lugar donde se produce la interacción entre la mente y el cerebro, este lugar ha de ser localizable. Sin embargo, las especulaciones de Descartes (él pensó que el lugar de la interacción se encontraba en la epífisis) fueron pronto refutadas. Tampoco se halló en el cerebro ningún otro lugar visible en el que el comportamiento de las neuronas sólo fuera explicable por la acción de un espíritu inmaterial. Dejando a un lado que en el cerebro no parece haber ningún "lugar" en el que se produzca una interacción, la cuestión del tipo de acción recíproca entre mente y cerebro permanece abierta. Algunos filósofos suponen que dicha interacción se produce por medio de efectos cuánticos. y mundo material. Para Descartes, la rex cogitans y la rex extensa eran dos entidades separadas, diferentes. El pensador francés divorció a la conciencia del cuerpo y describió a este último como una máquina. Desde el punto de vista cartesiano, es posible hablar del cuerpo como entidad humana, como algo propiamente humano: mente y materia son substancias diferentes e interactúan entre sí: materia y mente son entidades diferentes.

Los filósofos empiristas ingleses, Hume, Locke, Berkeley, por el contrario, reivindicarán en oposición al cartesianismo, el cuerpo como entidad que "siente" y es importante para que ejecute el acto de conocer, con su máxima: "Nada hay en el entendimiento que primero no haya pasado por los sentidos (sensus: sentir con el cuerpo), los empiristas darán cabida a otra faceta del modo de concebir lo corporal17.

En John Locke, el ser humano percibe las cosas concretas, las cualidades pero no el ser, no la sustancialidad. Dirá que la idea de sustancia tiene un contenido confuso, la necesitamos como soporte de las cualidades sensibles pero no conocemos "es un no sé qué". Aceptamos la existencia del yo captado por intuición. La de Dios que es la causa de nuestra existencia y la de los cuerpos materiales que son causa de nuestras sensaciones.

En George Berkeley no existen ideas abstractas, todo conocimiento es sensible. El ser del espíritu es percibido, por tanto, existe el yo o espíritu finito y Dios o espíritu infinito. Las cosas materiales sólo tienen sentido, sólo existen en cuanto son percibidas por un espíritu ya sea el alma ya sea Dios. No tienen realidad independiente, no son sustancias.

17 Los empiristas parten siempre de la experiencia y, por tanto, de la sensibilidad corporal. La experiencia es la fuente, pero también el límite de nuestro conocimiento, parte de que la realidad era sustancial para los racionalistas, esta sustancialidad se va perdiendo en los empiristas desde Locke a Hume, precisamente por basarlo todo en lo sensible, en la experiencia del cuerpo que capta por medio de los sentidos. 
En el pensamiento de David Hume, la base del conocimiento son las impresiones. Las ideas se derivan de las impresiones y son verdaderas si proceden directamente de ellas. Las impresiones sólo nos dan hechos que suceden. Nunca hay relación causal entre ellos. Esta relación es fruto de la elaboración mental, por tanto, creemos en la causalidad, no la conocemos, ya que no procede de ninguna impresión.

De igual forma se dieron, otras formas de dualismo, entre las que cabe destacar, el paralelismo psicofísico, desarrollado por Gottfried Leibniz (1988), quien afirmaba que espíritu y materia son dos substancias diferentes y que dichas substancias no interactúan entre sí.

Por otra parte el pensador francés Nicolás de Malebranche admite la esencial heterogeneidad de las dos substancias, cuerpo y espíritu. Malebranche resuelve la imposibilidad de una acción recíproca. El cuerpo extenso, capaz sólo de una acción mecánica, no puede originar las sensaciones en el alma inextensa, espiritual. Lo que sucede, según Malebranche, es que no son las cosas de este mundo -almas o cuerpos- las que actúan, sino que es Dios el solo autor de todo movimiento, sujeto único de causalidad eficiente. Dios pone de acuerdo -sincroniza- las dos substancias, produciendo en el alma sensaciones con ocasión de los hechos físicos, y movimientos en el cuerpo con ocasión de voliciones en el alma. De aquí el nombre de ocasionalismo con que se conoce a esta teoría.

Baruc de Spinoza ${ }^{18}$, heredero crítico del racionalismo cartesiano, propone una sola sustancia para todos los atributos. Este pensador holandés se declara monista, esto es, no cree en la existencia de un dualismo cuerpo-alma. Para Spinoza, el hombre es cuerpo y mente y todo en su conjunto es parte de una sustancia universal con infinitos modos e infinitos atributos, algo que

18 Spinoza, no cesa de asombrarse del cuerpo. No se asombra de tener un cuerpo, sino de lo que puede el cuerpo. $Y$ es que los cuerpos no se definen por su género o por su especie, por sus órganos y sus funciones, sino por lo que pueden, por los afectos de que son capaces, tanto en pasión como en acción (Spinoza 2003). da lugar a un "monismo neutral". Con su propuesta, no quiere convertir el cuerpo en un modelo, y el alma en una simple dependencia del cuerpo. Su empresa es mucho más sutil: eliminar la pseudosuperioridad del alma sobre el cuerpo, tanto el alma como el cuerpo, expresan una misma y única cosa: un atributo del cuerpo es también un sentido del alma.

\section{Época Contemporánea}

Con la aparición de los llamados "maestros de la sospecha", la filosofía da un viraje total en la forma de concebir al hombre, la historia, el mundo y, en general, a la sociedad. Es importante detenerse en algunas obras de Schopenhauer, Nietzsche y Freud para extractar sus conceptos sobre lo corporal y lo corpóreo.

En el caso de Nietzsche, José Lara en su libro Nietzsche, un pensador póstumo. El cuerpo como centro de gravedad (1998), matiza, además, que esta descalificación del cuerpo en ambos casos trajo como consecuencia que éste fuera abandonado como objeto de reflexión y análisis teóricos consecuentes. Ahora bien, de esta forma se abre un debate con respecto a la razón y a los vínculos que la unen inevitablemente con el espíritu humano. En todo caso, éste es un tema sobre el que Nietzsche va a profundizar. De hecho, su denuncia contra Platón y el cristianismo no tiene como objetivo principal negar la significación que ambos han tenido para Occidente, sino evaluar su significado y las consecuencias que tuvo para el pensamiento filosófico.

El pensamiento de Nietzsche, con referencia al cuerpo, va a tener acogida, entre otros, en el pensador antioqueño Fernando González. Para este pensador, el cuerpo no se da como una dualidad entre el alma y los sentidos, sino más bien como una unidad: "El espíritu se ha unificado con el cuerpo y con la tierra: todo es una unidad; no hay contradicción en nosotros; somos tan armoniosos como el amibo, el unicelular"(González, 1995, p. 108). Como lo pensaba Nietzsche en el "sí mismo", donde la razón y el alma son sólo una parte del cuerpo: "Detrás de tus 
pensamientos y sentimientos hermano mío, hay un amo más poderoso, un guía desconocido. Se llama 'sí mismo' habita en tu cuerpo; es tu cuerpo" (Nietzsche, 1999, p. 45).

Más adelante la fenomenología ${ }^{19}$, cuyo precursor en Francia fue Gabriel Marcel (1889-1973), había tematizado el problema de una "filosofía concreta", en el cual el tema del cuerpo, "mi" propio cuerpo, "tu cuerpo" estaban unidos a una reflexión ontológicaantropológica, ideas que provenían de Alemania y que fueron causando un cambio radical en la manera de entender al hombre como existencia corpórea. En este proceso Francia se beneficia con el éxodo de grandes pensadores provenientes del este de Europa: Georges Gurvitch, Aron Gurwitsch, Alexandre Kojéve, Alexandre Koyré y otros. Estos pensadores se encargarían de difundir nuevas ideas sobre el tema del hombre. Por otro lado, estaba el aporte de las ideas de Husserl, Scheler y Heidegger, además del creciente interés por las ideas de Freud y la teoría de la Gestalt.

A mitad del siglo pasado, Michel Henry (2007), procurando establecer el carácter concreto de la subjetividad frente al idealismo, defendió la tesis de que la subjetividad coincide con nuestro propio cuerpo y se apoya en la ontología de Maine de Biran (1766-1824), uno de los pensadores franceses más originales y menos conocidos. Henry considera que de Biran fue el único capaz de plantear la posibilidad del cuerpo como apercepción inmediata interna, por lo que se posecionó frente a la fenomenología francesa y alemana, para las cuales la vida se caracteriza primeramente

19 La corriente fenomenológico-existencial del cuerpo, introduce fundamentalmente tres principios para la constitución de la actual antropología filosófica. En primer lugar, la superación de la idea sustancialista de la realidad del cuerpo, que domina -mutatis mutandis- en el dualismo clásico y particularmente moderno. En segundo lugar, la afirmación de la conciencia corporal como subjetividad auténtica y concreta, y de su rol fundamental en la constitución de las estructuras del mundo humano. Y, en tercer lugar, la posibilidad de una comprensión ontológica del ser del hombre por su condición encarnada. La filosofía fenomenológicoexistencial del cuerpo propio significa, en último análisis, una original y concreta forma de acceso a la dimensión propiamente metafísica de la experiencia humana como intencionalidad y trascendencia. Con todo, la vida procede de la subjetividad que brota del pathos inmediato de la propia corporeidad, incluso antes de haber recibido toda representación. El trabajo de Henry con base en la ontología de de Biran, ofrece la visión de una voluntad de la vida que se obstina "por seguir viviendo" y la revelación en cada ser humano de la propia vida como autodonación radical y primera que se experimenta en el cuerpo.

Es importante recordar que en Francia la fenomenología, a diferencia de Alemania, estaba en diálogo y en contacto permanente con el existencialismo, enfrentando al marxismo hegeliano. Dicha fenomenología francesa tiene como característica común que ofrece la fórmula de una fenomenología existencial que agrega nuevas variantes a las versiones trascendental y ontológica, y que muestra un parentesco con la hermenéutica del Dassein de Heidegger. La fenomenología francesa también surge como un método específico sobre una temática concreta: la temática de la existencia. Esta "nueva forma de fenomenología existencial" entre los pensadores franceses adquiere unos rasgos distintivos: el cuerpo, la corporalidad, la libertad y los otros ${ }^{20}$.

Específicamente, el cuerpo y la corporalidad constituyen el medio constante para la relación con los otros, con el mundo y en especial consigo mismo. Entre los pensadores que más directamente trabajaron el tema del cuerpo están Merleau-Ponty, Emmanuel Mounier y Michel Foucault, quienes asumen el trabajo sobre el cuerpo y la corporalidad desde estos presupuestos:

\footnotetext{
20 El problema fenomenológico-existencial sobre el cuerpo, que se inicia en esta época, fundamentalmente, aporta tres principios para la constitución de la actual antropología filosófica: la superación de la idea sustancialista de la realidad del cuerpo, que domina en el dualismo clásico y particularmente moderno. La afirmación de la conciencia corporal como subjetividad auténtica y concreta, y de su rol fundamental en la constitución de las estructuras del mundo humano y, por último, la posibilidad de una comprensión ontológica del ser del hombre por su condición encarnada. La filosofía fenomenológico-existencial del cuerpo propio significa, en último análisis, una original y concreta forma de acceso a la dimensión propiamente metafísica de la experiencia humana. (Mainetti, 1972
} 
- Conciben el cuerpo como límite: hay en el cuerpo un acercamiento a la fragilidad, al sometimiento de las fuerzas que ordenan en lo físico y lo biológico. A su vez, esa conciencia del límite le permite al hombre acercarse a una nueva comprensión del mundo que otros han entendido por él y que él mismo en este momento se ha permitido entender para sí mismo.

- Reflexionan sobre el cuerpo ligado al tiempo y al espacio, y este reconocimiento permite al hombre la posibilidad de autocomprensión y la comprensión de lo que le rodea. Merlau-Ponty, desde el análisis de la percepción, y Foucault, desde la reflexión del cuidado de sí mismo, intentan una nueva lectura del hombre como ser corpóreo que se trasciende en el tiempo y el espacio.

- Analizan el cuerpo como principio de no-instrumentalidad: a través de su propio cuerpo el hombre tiene, hasta cierto punto, dominio sobre las fuerzas de la naturaleza. Posee actividad y dinámica. No es instrumento porque el instrumento se refiere a algo que está fuera de mi alcance y es susceptible de "manipular"; éste no es el caso del cuerpo. Por el contrario, a partir de él se genera su conocimiento del mundo.

- Evocan al cuerpo como lenguaje: puede a través del lenguaje participar activa y solidariamente en la transformación de su entorno, ya que por medio de la palabra se orienta hacia la co-construcción del mundo que le rodea. Las diversas partes del cuerpo, participan de forma diversa en la construcción del lenguaje, pues en el fondo del lenguaje no hace sino desarrollar y especificar el lenguaje fundamental que es el propio cuerpo.

- El cuerpo como presencia: en las relaciones con los demás, el cuerpo está presente, es presencia del hombre mismo. Todo cuerpo en cuanto presencia está referido a los demás y a su vez se autocontiene a sí mismo. En cierta forma está unido a los demás y a la vez se diferencia de los demás: se percibe "un cuerpo" diferente a "otro cuerpo", aunque ambos pertenecen al género humano.

En la actualidad, Jean Luc Nancy (2001), profesor de filosofía de la Universidad de Estrasburgo, en su obra Corpus, publicada en Francia en 1992, desarrolla su filosofía del cuerpo. Algunos consideran que Nancy desarrolla una nueva ontología, en este caso una ontología del cuerpo. Su tesis de que no tenemos un cuerpo, sino que, más bien, lo somos como exterioridad y exposición infinita, como cuerpo volcado hacia fuera, se asemeja a los planteamientos de Merleau-Ponty. Concretamente, con relación al tema del trasplante de órganos, este pensador francés propone una discusión en torno al estatuto ontológico y epistémico del cuerpo y las prácticas médicas asociadas a las experiencias traumáticas y límites de éste.

De muchas formas, los planteamientos de Nancy van más allá de una reflexión de corte teleológicobiológico para entrar en el campo del cuerpo como "acontecimiento"; esto implica dejar de concebir al cuerpo como algo "organizado" sobre la base de una finalidad separada de sí mismo, ya sea que le trascienda o le anteceda. Ya no se podrá hablar de finalidades en función de un cuerpo posorgánico o inorgánico que se encuentra direccionado a un fin trascendente, sino que lo que acontece, sucede como evento determinado en sí mismo (Vásquez, s.f.). El cuerpo es un objeto dado a un pensamiento finito; la verdad del sujeto es su exterioridad y su excesividad: su exposición infinita, el cuerpo volcado hacia fuera:

Soy la enfermedad y la medicina, soy la cancerosa y el órgano trasplantado, soy los agentes inmunodepresores y sus paliativos, soy los ganchos de hilo de acero que me sostienen el esternón y soy ese sitio de inyección cosido permanentemente bajo la clavícula, así como ya era, por otra parte, esos clavos en la cadera y esa placa en la ingle (Nancy, 2006).

Se puede advertir que esta idea gravita alrededor del discurso de Derrida sobre la diferencia: la diferencia 
entre el pensamiento y el cuerpo, entre forma y contenido.

Nancy crea un concepto de cuerpo que se opone a la idea platónica del cuerpo como cárcel del alma. La idea e imagen de un contenedor da paso a una metáfora de la deconstrucción orgánica -en este caso del texto, a través de la cual Nancy no quiere escribir del o sobre el cuerpo, sino quiere escribir e inscribir el cuerpo. Escribir el cuerpo significa hacer inscripciones sobre él, tocarlo y esculpirlo con el pensamiento, desarrollar una somato-grafía, para hacer que el cuerpo mismo sea leído.

\section{Síntesis}

Como síntesis de las reflexiones anteriores, se puede decir que las interpretaciones más sobresalientes sobre el cuerpo han sido las siguientes:

- La platónica-patrística: claramente dualista, en la cual el cuerpo y el alma son dos realidades unidas exteriormente; durante la existencia terrena del hombre se hace énfasis en la significación negativa de la condición corpórea.

- La aristotélico-tomista: pone el acento en la estructura ontológica del ser humano. El hombre es unidad, no como producto de la conjunción de dos realidades diversas, sino de dos principios correlativos: materia y forma. Se acentúa el aspecto positivo del cuerpo frente a la apreciación negativa de la anterior interpretación.

- La racionalista: postulada por Descartes, en la que se establece una división radical entre el cuerpo y la conciencia. El hombre es más identificado con la conciencia, por su naturaleza racional, ella está ligada y unida con el cuerpo, pero no es idéntica a él. El cuerpo humano se explica sobre la base del movimiento mecánico de los átomos, en este caso, es concebido como una realidad atómica, física y extensa.
- La mecanicista: lleva hasta las últimas consecuencias la interpretación atomista del cuerpo introducida por Descartes. El mundo es un conjunto de fuerzas mecánicas, el cuerpo no se escapa de esta interpretación, sino que hace parte de ella.

- $\quad$ La materialista: reduce todas las expresiones humanas a la materia corpórea. Fuera de ella, nada se entiende y el hombre como ser que posee cuerpo "material", no puede rebasar los límites de la misma materia.

- La fenomenológica-existencialista: el ser humano, no "tiene un cuerpo", sino que "es su cuerpo", como exterioridad y exposición infinita, como presencia en el mundo.

Estas interpretaciones se mueven en torno a un mismo problema: ¿qué papel cumple el cuerpo en la existencia del hombre?, o también ¿es el cuerpo un elemento fundamental para la comprensión de la esencia humana? Algunos pensadores contemporáneos van a afirmar cómo la idea de corporeidad en la sociedad actual ha cambiado y ha terminado con el binomio cuerpo y mente, constituyéndose así, en el "sujeto en sí y para sí", pues nada importa más que el cuerpo como fuente de sensaciones, placeres y éxito.

El pensador posmoderno Gilles Lipovetsky (2000), afirma que el ser humano contemporáneo, reclama juventud y un no envejecimiento; el hedonismo, el consumo de promesas de felicidad, la evasión son sinónimos de una sociedad que fomenta un tipo de felicidad humana basada en el éxito del cuerpo; debido a esto, los cuidados hacia el cuerpo crecen, de allí que se recurra a cosas como la moda (Lipovetsky, 1987), para reafirmar el presente del cuerpo que se tiene y a la vez se exhibe; pero la realidad es que día a día se multiplica la ansiedad y la insatisfacción. Así que ahora, el concepto de felicidad es el más irónico, pues las recriminaciones y las insatisfacciones del ser humano consigo mismo, le hacen evidenciar un divorcio interior profundo, en el que sigue prevaleciendo el culto a los ídolos. En esta sociedad, caracterizada 
por una cultura de masas, según el pensador francés, el cuerpo cumple un papel primordial, pues sigue estando presente en el aquí y ahora del individuo.

Para terminar, una cita de Lipovetsky, en "Le bonheur paradoxal, essai sur la société d'hyperconsommation", que ilustra esta conclusión:

"L'hédonisme, la consommation promet des bonheurs, de l'évasion, c'est une société qui stimule une marche au bonheur dans ses référentiels, mais la réalité c'est que l'on voit la multiplicité des anxiètés, la morosité, l'inquiétude, le ras le bol, l'insatisfaction quotidienne. Donc voilà, l'idée de bonheur paradoxal est: que plus la société marche au bonheur plus montent les plaintes, les récriminations, les insatisfactions"(Lipovetsky, 2006).

\section{Referencias}

Aquino, T. (1988). Suma Teológica (8ª edición. I, C. 76 , a. 1). Madrid: BAC.

Chalmers, D. (1999). La mente consciente: en busca de una teoría fundamental. Editorial Gedisa.

Churchland, P. (1992). Materia y conciencia: Introducción contemporánea a la filosofía de la mente. Editorial Gedisa.

Davidson, D. (1992). Mente, mundo y acción. Barcelona: Ediciones Paidós Ibérica.

Etienne, G. (1922). La philosophie au moyen-âge (vol. II: De saint Thomas d'Aquin à Guillaume d'Occam). París: Payot. Flammarion.

Foucault, M. (1990). Tecnologías del Yo y otros textos afines. Barcelona: Ediciones Paidós-Ibérica.

Foucault, M. (1998). Hermenéutica del sujeto. BarceIona: Ediciones la Piqueta.

Foucault, M. (2006). Un Cours Inédit, Lección sobre la Ilustración. Barcelona: Ediciones Paidós-Ibérica.
González, F. (1995). Viaje a pie. Medellín: Editorial Bedout.

Henry, M. (2007). Filosofía y fenomenología del cuerpo: ensayo sobre la ontología de Maine de Biran. Barcelona: Ediciones Sígueme.

Lara, J. (1998). Nietzsche, un pensador póstumo. El cuerpo como centro de gravedad, Barcelona: Anthropos.

Lipovetsky, G. (1987). El Imperio de lo efímero: la moda y su destino en las sociedades modernas. Anagrama.

Leibniz, G. (1988). Monadología, Barcelona: Paidós.

Lipovetsky, G. (2000). La era del vacío: ensayos sobre el individualismo contemporáneo (13 a edición). Anagrama.

Lipovetsky, G. (2006). Le bonheur paradoxal, essai sur la société d'hyperconsommation. París: Editions Gallimard.

Merleau-Ponty, M. (1984). Fenomenología de la percepción. Barcelona: Editorial Planeta.

Mounier, E. (1996). El personalismo. Bogotá: Editorial Nueva América.

Nancy, J.L. (2000). El intruso. París: Éditions Galilée.

Nancy, J.L. (2001). Corpus. Nápoles: Editorial Cronopio.

Nicholl, C. (2005). El vuelo de la mente. México D.F.: Ediciones Taurus.

Nietzsche, F. (1999). Así habló Zaratustra. Bogotá: Ediciones Universales.

Popper, K.R. \& Eccles, J. (1985). El yo y su cerebro. Madrid: Labor. 
Pouchelle, M.C. (1983). Corps et chirurgie á l'apogée du Moyen-Age. París.

Putnam, H. (2001). La trenza de tres cabos: la mente, el cuerpo y el mundo. Madrid: Siglo XXI.
Spinoza, B. (2003). Obras completas. Madrid: Alianza Editorial. Spinoza.

Turing, A. (1985). Mentes y máquinas. Madrid: Editorial Tecnos. 